\title{
Studies on Application of Amino Acid as Medicinal Agent. I. Syntheses of Amino-tert-alcohol Derivatives
}

\author{
Seigoro Hayashi, Mitsuru Furukawa, Yoko Fujino, $\left.{ }^{1} a\right)$ \\ Naoto Matsuishi ${ }^{1 b}$ ) and Tadashi OhKawara ${ }^{1} c$ ) \\ Faculty of Pharmaceutical Sciences, Kumamoto University, ${ }^{1 a)}$ Tokyo \\ Tanabe Seiyaku Co., Ltd.1b) and Tanabe Seiyaku Co., Ltd.1c)
}

(Received June 4, 1968)

\begin{abstract}
In order to find non-narcotic analgesics, 54 compounds of amino-tert-alcohol, in which a variety of substituent groups were involved at the carbon atom in 1-position and at the amino group, were synthesized by reacting various $\alpha$ - and $\beta$-amino acid esters with Grignard reagents. These amino acid esters were readily reacted with alkyl Grignard reagents to give the expected compounds. However, when bulky aromatic Grignard reagents were submitted to react, the reactions were found to stop at the stage of intermediate formation of the corresponding ketones.
\end{abstract}

A variety of amino-alcohols having various alkyl substituents at the carbon atom in 1-position, which contain a primary amino group, have been synthesized by Kanao, ${ }^{2}$ ) and some of them were found to have a local anesthetic activity. This finding seemed to suggest that the alkyl substituents at the carbon atom in 1-position might contribute to the generation of the anesthetic activity. Though a number of studies for analgesics have hitherto been widely carried out, no excellent non-narcotic analgesics have been found yet. Thus, for the purpose of finding new effective non-narcotic analgesics, a number of substituted aminotert-alcohols containing a variety of substituents at the carbon atom in 1-position and at the amino group, having the following general formula, in which $R_{1}, R_{2}$ and $R_{3}$ stand for hydrogen atom, alkyl or aryl group, were attempted to synthesize.

$$
\mathrm{R}_{1} \mathrm{R}_{2} \mathrm{~N}\left(\mathrm{CH}_{2}\right)_{n} \mathrm{C}\left(\mathrm{R}_{3}\right)_{2} \mathrm{OH} \quad(n=1,2)
$$

In order to prepare these compounds, various natural and synthetic $\alpha$ - and $\beta$-amino acid esters were allowed to react with a variety of Grignard reagents. Especially, the use of natural amino acids as materials implies to apply them as medical drugs. Synthetic $\alpha$ - and $\beta$-amino acid esters, ${ }^{3 a, b, c, d, e)}$ in which various amino groups, such as primary amino, methylamino, ethylamino, dimethylamino, diethylamino, benzylamino, pyrrolidino, piperidino, morpholino and piperazino groups, were involved in the structure, were prepared by treatment of ethyl chloroacetate or carboxylic acid esters containing a double bond, such as acrylic acid, crotonic acid and cinnamic acid esters, with corresponding amines by means of several methods. Grignard reagents were prepared by treating alkyl or aryl halogenides with magnesium in absolute ether by the usual manner. To the etheral solution of these Grignard reagents thus prepared was added the free base or the hydrochloride of these amino acid esters, followed by mild reflux for 30 minutes. Then the reaction mixture was allowed to be hydrolyzed with dilute hydrochloric acid. The resulting by-products separated were removed by extraction with ether and the aqueous layer was made alkaline with ammonium hydroxide. The separated oily product was extracted with ether and purified by distillation or recrystal-

1) Location: a) Oe-hon-machi, Kumamoto; b) Itabashi, Tokyo; c) Higashiyodogawa, Osaka.

2) S. Kanao and K. Shinozuka, Yakugaku Zasshi, 50, 1155 (1930).

3) a) C.A. Bischoff, Chem. Ber., 31, $2840(1898)$; b) D.W. Adamson, J. Chem. Soc., 1949, 144; c) B. Flürscheim, J. Prakt. Chem., 68, 350 (1903); d) K. Morsch, Monatsch, 60, 50 (1932); e) H. Pacheo, D. Dreux and A. Beuillian, Bull. Soc. Chem. (France), 1379 (1962); C.A., 58, 7933 (1963). 
Table I.

$\mathrm{R}^{\prime}$
$\mathrm{R}_{2} \mathrm{~N}-\mathrm{CH}_{2}-\mathrm{C}-\mathrm{OH}$
1
$\mathrm{R}^{\prime}$

\begin{tabular}{|c|c|c|c|c|c|c|c|}
\hline \multirow[b]{2}{*}{$\begin{array}{l}\text { Compd. } \\
\text { No. }\end{array}$} & \multirow[b]{2}{*}{$\mathrm{R}_{2} \mathrm{~N}$} & \multirow[b]{2}{*}{$\mathrm{R}^{\prime}$} & \multirow[b]{2}{*}{$\begin{array}{c}\mathrm{bp} \text { or } m p \\
\left({ }^{\circ} \mathrm{C} / \mathrm{mm}\right)\left({ }^{\circ} \mathrm{C}\right)\end{array}$} & \multirow{2}{*}{$\begin{array}{c}\text { Yield } \\
(\%)\end{array}$} & \multirow[b]{2}{*}{ Formula } & \multicolumn{2}{|c|}{ Analysis (\%) } \\
\hline & & & & & & Calcd. & Found \\
\hline
\end{tabular}

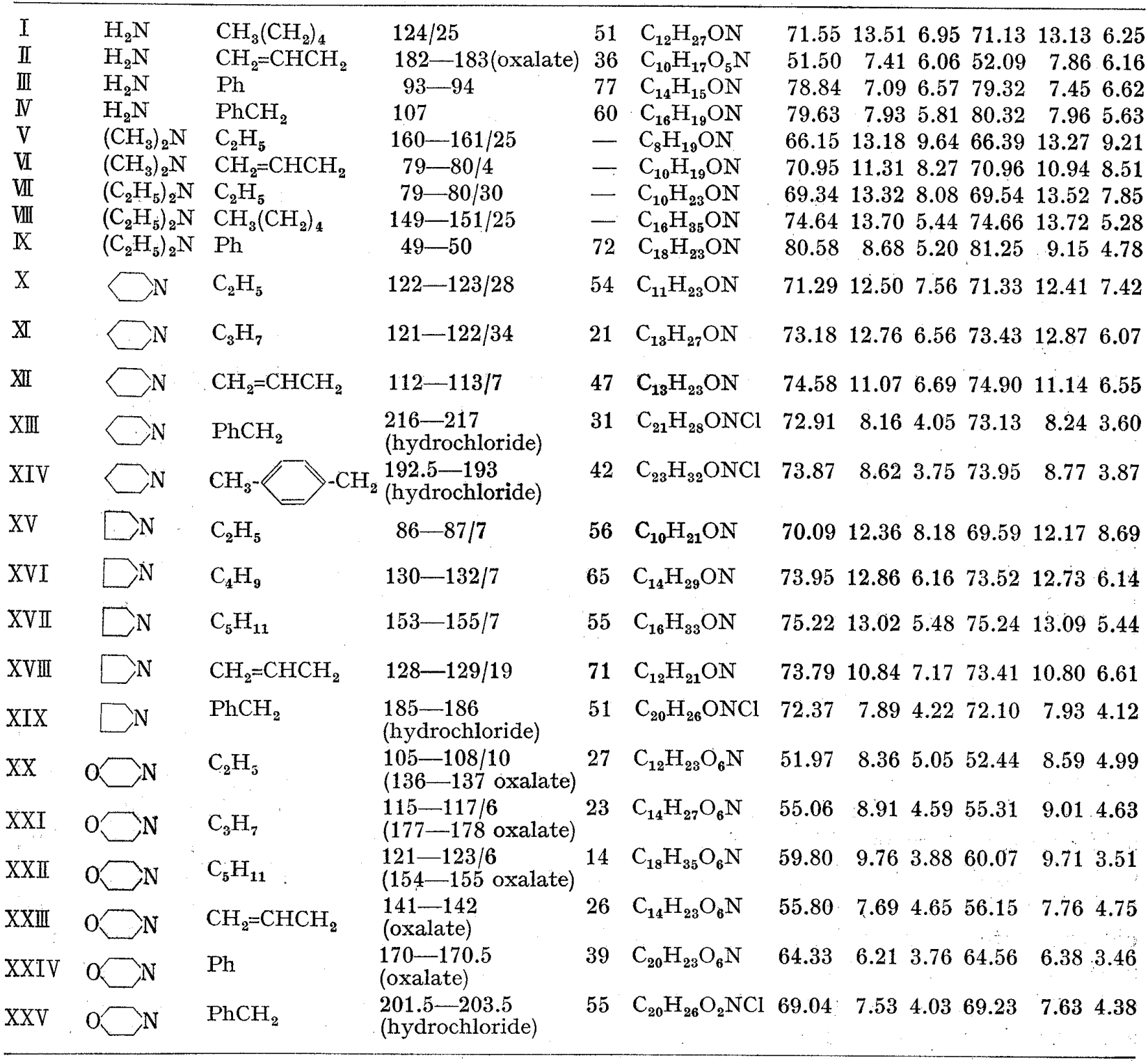

$\begin{array}{cc}\mathrm{H}_{2} \mathrm{~N} & \mathrm{R} \\ \mathrm{PhCH}_{2}-\mathrm{CH}-\mathrm{C}-\mathrm{OH} \\ \stackrel{1}{\mathrm{R}}\end{array}$

\begin{tabular}{|c|c|c|c|c|c|c|c|c|c|}
\hline \multirow{3}{*}{$\begin{array}{l}\text { Compd. } \\
\text { No. }\end{array}$} & \multirow{3}{*}{$\mathrm{R}$} & \multirow{3}{*}{$\begin{array}{c}\mathrm{bp} \text { or } \operatorname{mp} \\
\left({ }^{\circ} \mathrm{C} / \mathrm{mm}\right)\left({ }^{\circ} \mathrm{C}\right)\end{array}$} & \multirow{3}{*}{$\begin{array}{l}\text { Yield } \\
(\%)\end{array}$} & \multirow{3}{*}{ Formula } & \multicolumn{5}{|c|}{ Analysis (\%) } \\
\hline & & & & & \multicolumn{3}{|c|}{ Calcd. } & \multicolumn{2}{|r|}{ Found } \\
\hline & & & & & $\mathrm{C}$ & $\mathrm{H}$ & $\mathrm{N}$ & c & $\mathrm{H} \therefore \mathrm{N}$ \\
\hline $\begin{array}{l}\text { XXVI } \\
\text { XXVII }\end{array}$ & $\mathrm{C}_{2} \mathrm{H}_{5}$ & $115-117 / 4(184-185$ oxalate $)$ & 27 & $\mathrm{C}_{15} \mathrm{H}_{23} \mathrm{O}_{5} \mathrm{~N}$ & 60.50 & 7.80 & 4.71 & 60.85 & $7.73 \cdot 5.13$ \\
\hline & $\mathrm{C}_{3} \mathrm{H}_{7}$ & $202.5-203$ (oxalate) & 10 & $\mathrm{C}_{19} \mathrm{H}_{27} \mathrm{O}_{5} \mathrm{~N}$ & 62.74 & 8.36 & 4.31 & 62.79 & $8.55 \quad 4.24$ \\
\hline
\end{tabular}


XXVIII $\quad \mathrm{CH}_{2}=\mathrm{CHCH}_{2} \quad 199-199.5$ (oxalate) XXIX $\mathrm{Ph} \quad 255-256$ (hydrochloride) XXX $\quad \mathrm{PhCH}_{2} \quad 139-140$ (hydrochloride)

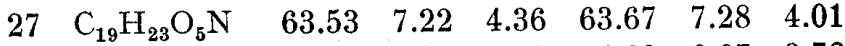

$\begin{array}{llllllll}28 & \mathrm{C}_{21} \mathrm{H}_{22} \mathrm{ONCl} & 74.21 & 6.52 & 4.12 & 74.62 & 6.67 & 3.78\end{array}$

$\begin{array}{llllllll}33 & \mathrm{C}_{23} \mathrm{H}_{26} \mathrm{ONCl} & 75.25 & 7.14 & 3.82 & 74.83 & 7.24 & 3.97\end{array}$

\begin{tabular}{|c|c|c|c|c|c|c|c|c|c|c|c|}
\hline \multirow{3}{*}{$\begin{array}{c}\text { Compd. } \\
\text { No. }\end{array}$} & \multirow{3}{*}{$\mathrm{R}_{2} \mathrm{~N}$} & \multirow{3}{*}{$\mathrm{R}^{\prime}$} & \multirow{3}{*}{$\begin{array}{l}\mathrm{bp} \text { or } \\
\left({ }^{\circ} \mathrm{C} / \mathrm{mm}\right) \\
\left({ }^{\circ} \mathrm{C}\right)\end{array}$} & \multirow{3}{*}{$\begin{array}{l}\text { Yield } \\
(\%)\end{array}$} & \multirow{3}{*}{ Formula } & \multicolumn{6}{|c|}{ Analysis (\%) } \\
\hline & & & & & & \multicolumn{3}{|c|}{ Calcd. } & \multicolumn{3}{|c|}{ Found } \\
\hline & & & & & & $\mathrm{C}$ & $\mathrm{H}$ & $\mathrm{N}$ & $\mathrm{C}$ & $\mathrm{H}$ & $\mathrm{N}$ \\
\hline XXXI & $\left(\mathrm{C}_{2} \mathrm{H}\right.$ & $\mathrm{C}_{2} \mathrm{H}_{5}$ & $74-75 / 7$ & 22 & $\mathrm{C}_{11} \mathrm{H}_{25} \mathrm{ON}$ & 70.53 & 13.45 & 7.48 & 70.05 & 12.94 & 7.73 \\
\hline XXXII & $\left(\mathrm{C}_{2} \mathrm{H}_{5}\right)_{2} \mathrm{~N}$ & $\mathrm{C}_{3} \mathrm{H}_{7}$ & $108-109 / 9$ & 25 & $\mathrm{C}_{13} \mathrm{H}_{27} \mathrm{ON}$ & 72.49 & 13.57 & 6.51 & 72.64 & 13.62 & 6.67 \\
\hline XXXIII & $\left(\mathrm{C}_{2} \mathrm{H}_{5}\right)_{2} \mathrm{~N}$ & $\mathrm{CH}_{2}=\mathrm{CHCH}_{2}$ & $92-93 / 3$ & 64 & $\mathrm{C}_{13} \mathrm{H}_{25} \mathrm{ON}$ & 74.88 & 11.92 & 6.63 & 73.66 & 11.93 & 6.78 \\
\hline XXXIV & $\left(\mathrm{C}_{2} \mathrm{H}_{5}\right)_{2} \mathrm{~N}$ & $\mathrm{Ph}^{a)}$ & $207-209$ (oxalate) & 52 & $\mathrm{C}_{21} \mathrm{H}_{27} \mathrm{O}_{5} \mathrm{~N}$ & 67.54 & 7.29 & 3.76 & 67.95 & 7.43 & 3.90 \\
\hline XXXV & $\left(\mathrm{C}_{2} \mathrm{H}_{5}\right)_{2} \mathrm{~N}$ & $\mathrm{PhCH}$ & $40-41$ & 39 & $\mathrm{C}_{21} \mathrm{H}_{29} \mathrm{ON}$ & 80.98 & 9.39 & 4.50 & 81.13 & 9.42 & 4.57 \\
\hline XXXVI & $\left(\mathrm{C}_{2} \mathrm{H}_{5}\right)_{2} \mathrm{~N}$ & $\mathrm{CH}_{3}-$ & $172-174 / 6$ & 37 & $\mathrm{C}_{23} \mathrm{H}_{33} \mathrm{ON}$ & 81.36 & 9.80 & 4.12 & 81.34 & 9.94 & 4.49 \\
\hline XXXVII & (CH & $\mathrm{C}_{2} \mathrm{H}_{5}$ & $57-59 / 4$ & 22 & $\mathrm{C}_{9} \mathrm{H}_{21} \mathrm{ON}$ & 67.87 & 12.29 & 8.80 & 67.57 & 13.30 & 8.92 \\
\hline XXXVIII & $(\mathrm{CH}$ & $\mathrm{C}_{4} \mathrm{H}_{9}$ & $117-118 / 13$ & 18 & $\mathrm{C}_{13} \mathrm{H}_{29} \mathrm{ON}$ & 72.49 & 13.57 & 6.51 & 72.30 & 13.55 & 6.65 \\
\hline XXXIX & $\mathrm{CH}$ & $\mathrm{C}_{5} \mathrm{H}_{11}$ & $125 / 4$ & 44 & $\mathrm{C}_{15} \mathrm{H}_{33} \mathrm{ON}$ & 74.00 & 13.66 & 5.75 & 74.24 & 13.62 & 5.88 \\
\hline $\mathrm{XL}$ & $\left(\mathrm{CH}_{3}\right.$ & $\mathrm{CH}_{2}=\mathrm{CHCH}_{2}$ & $79-80 / 5$ & 65 & $\mathrm{C}_{11} \mathrm{H}_{21} \mathrm{ON}$ & 72.08 & 11.55 & 7.64 & 71.87 & 11.63 & 7.67 \\
\hline XLI & (CH & $\mathrm{PhCH}_{2}$ & $\begin{array}{c}195-198 / 8 \\
63-64\end{array}$ & 38 & $\mathrm{C}_{19} \mathrm{H}_{25} \mathrm{ON}$ & 80.51 & 8.89 & 4.94 & 80.43 & 8.84 & 5.23 \\
\hline XLII & $(\mathrm{CH}$ & 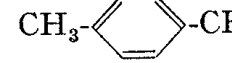 & $185-187 / 3$ & 41 & $\mathrm{C}_{21} \mathrm{H}_{29} \mathrm{ON}$ & 80.98 & 9.39 & 4.50 & 80.81 & 9.24 & 4.59 \\
\hline XLIII & & $\mathrm{CH}_{2}=\mathrm{CHCH}_{2}$ & $121-122 / 5$ & 78 & $\mathrm{C}_{14} \mathrm{H}_{25} \mathrm{ON}$ & 75.28 & 11.28 & 6.27 & 75.17 & 11.47 & 6.35 \\
\hline XLIV & & $\mathrm{PhCH}_{2}$ & $\begin{array}{c}170-176 / 7 \\
49-50\end{array}$ & 37 & $\mathrm{C}_{22} \mathrm{H}_{29} \mathrm{ON}$ & 81.68 & 9.04 & 4.33 & 81.68 & 8.99 & 4.40 \\
\hline $\mathrm{XLV}$ & & $\mathrm{CH}_{2}=\mathrm{CHCH}_{2}$ & $139-142 / 7$ & 35 & $\mathrm{C}_{13} \mathrm{H}_{23} \mathrm{O}_{2} \mathrm{~N}$ & 69.29 & 10.29 & 6.22 & 69.20 & 10.30 & 6.18 \\
\hline XLVI & & $\mathrm{Ph}^{a)}$ & $\begin{array}{l}207-208 \\
\text { (hydrochloride) }\end{array}$ & 30 & $\mathrm{C}_{19} \mathrm{H}_{24} \mathrm{O}_{2} \mathrm{NCl}$ & 68.35 & 7.24 & 4.19 & 68.05 & 7.12 & 4.28 \\
\hline XLVII & & $\mathrm{PhCH}_{2}$ & $\begin{array}{l}208-209 \\
\text { (hydrochloride) }\end{array}$ & 55 & $\mathrm{C}_{21} \mathrm{H}_{30} \mathrm{O}_{2} \mathrm{NCl}$ & 69.69 & 7.80 & 3.87 & 70.10 & 7.86 & 4.04 \\
\hline XLVIII & & $\mathrm{C}_{4} \mathrm{H}_{9}$ & $125-126 / 4$ & 53 & $\mathrm{C}_{15} \mathrm{H}_{31} \mathrm{ON}$ & 74.62 & 12.94 & 5.80 & 74.52 & 13.17 & 5.58 \\
\hline XLIX & & $\mathrm{CH}_{2}=\mathrm{CHCH}_{2}$ & $117-118 / 3$ & 46 & $\mathrm{C}_{13} \mathrm{H}_{23} \mathrm{ON}$ & 74.58 & 11.07 & 6.69 & 74.21 & 11.08 & 6.52 \\
\hline L & & $\mathrm{PhCH}_{2}$ & $\begin{array}{l}207-208 \\
\text { (hydrochloride) }\end{array}$ & 53 & $\mathrm{C}_{21} \mathrm{H}_{28} \mathrm{ONCl}$ & 72.87 & 7.87 & 4.05 & 72.67 & 8.28 & 4.02 \\
\hline $\mathrm{LI}$ & $\mathrm{PhCH}_{2} \mathrm{NH}$ & $\mathrm{C}_{2} \mathrm{H}_{5}$ & $215-216$ (oxalate) & 12 & $\mathrm{C}_{16} \mathrm{H}_{25} \mathrm{O}_{5} \mathrm{~N}$ & 61.71 & 8.09 & 4.50 & 60.69 & 7.74 & 4.54 \\
\hline LII & $\mathrm{N} N \mathrm{~N}$ & $\mathrm{PhCH}_{2}$ & $\begin{array}{l}242-244 \text { (decomp } \\
\text { (hydrochloride) }\end{array}$ & .) 19 & $\mathrm{C}_{38} \mathrm{H}_{48} \mathrm{O}_{2} \mathrm{~N}_{2} \mathrm{Cl}_{2}$ & 71.79 & 7.61 & 4.41 & 71.80 & 7.64 & 4.41 \\
\hline
\end{tabular}

$$
\begin{array}{cc}
\mathrm{R}^{\prime} & \mathrm{R}^{\prime \prime} \\
\mathrm{R}_{2} \mathrm{~N}-\mathrm{C} \mathrm{C}-\mathrm{CH}_{2}-\mathrm{C}-\mathrm{C}-\mathrm{OH} \\
& \stackrel{1}{\mathrm{R}^{\prime \prime}}
\end{array}
$$

\begin{tabular}{|c|c|c|c|c|c|c|c|c|c|c|c|c|}
\hline \multirow{3}{*}{$\begin{array}{c}\text { Compd. } \\
\text { No. }\end{array}$} & \multirow{3}{*}{$\mathrm{R}_{2} \mathrm{~N}$} & \multirow{3}{*}{$\mathrm{R}^{\prime}$} & \multirow{3}{*}{$\mathrm{R}^{\prime \prime}$} & \multirow{3}{*}{$\underset{\left({ }^{\circ} \mathrm{Cp} / \mathrm{mm}\right)}{\left({ }^{\circ} \mathrm{C}\right)}$} & \multirow{3}{*}{$\begin{array}{l}\text { Yield } \\
(\%)\end{array}$} & \multirow{3}{*}{ Formula } & \multicolumn{6}{|c|}{ Analysis (\%) } \\
\hline & & & & & & & \multicolumn{3}{|c|}{ Calcd. } & \multicolumn{3}{|c|}{ Found } \\
\hline & & & & & & & $\mathrm{C}$ & $\mathrm{H}$ & $\mathbf{N}$ & $\mathrm{C}$ & $\mathrm{H}$ & $\mathbf{N}$ \\
\hline LIII & $\left(\mathrm{C}_{2} \mathrm{H}_{5}\right)_{2} \mathrm{~N}$ & $\mathrm{CH}_{3}$ & $\mathrm{CH}_{2}=\mathrm{CHCH}_{2}$ & $96-98 / 4$ & 38 & $\mathrm{C}_{14} \mathrm{H}_{27} \mathrm{ON}$ & 74.61 & 12.08 & 6.22 & 74.43 & 12.17 & 6.35 \\
\hline LIV & $\square_{N}$ & $\mathrm{Ph}$ & $\mathrm{PhCH}_{2}$ & $104--105$ & 42 & $\mathrm{C}_{27} \mathrm{H}_{31} \mathrm{ON}$ & 84.09 & 8.11 & 3.62 & 84.25 & 8.27 & 3.48 \\
\hline
\end{tabular}

a) These compounds were known. 


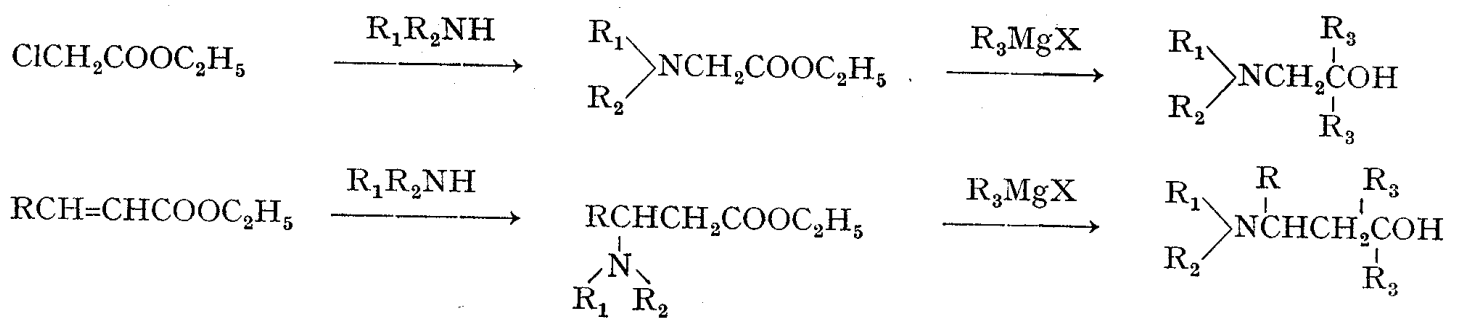

lization. When Grignard reagents containing comparative bulky substituents, such as amyl, benzyl, phenyl, $p$-xylyl and naphthyl, were employed in the reaction, the corresponding amino alcohols were directly isolated from the reaction mixture in the form of crystals of the hydrochloride in the course of this procedure for their slight solubility. Generally, aliphatic alkyl Grignard reagents were reacted readily with amino acid esters to give the corresponding amino alcohols. However, when aromatic Grignard reagents were submitted to react with amino acid esters, the reaction was stopped at the stage of the intermediate formation of ketones and the further reaction was not able to proceed, probably because of the increasing steric bulkiness due to aromatic ring.

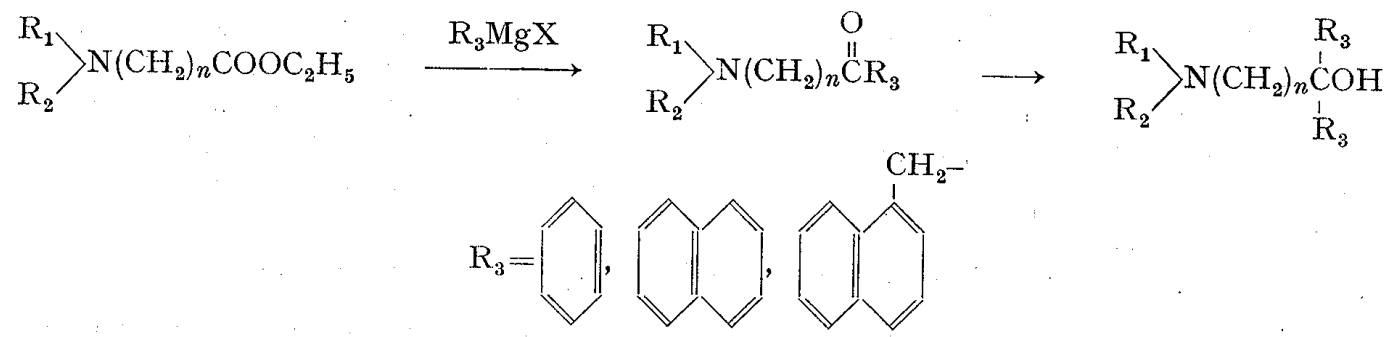

All of these amino-tert-alcohols synthesized were summarized in Table I.

The pharmacological activities of these compounds "will be reported in the other paper.

\section{Experimental}

$\boldsymbol{\alpha}$-Amino Acid Esters__ L-Phenylalanine Ithyl Ester Hydrochloride: It was prepared by the method of Fischer-Schodler ${ }^{4}$ using $30 \mathrm{~g}(0.184 \mathrm{~mole})$ of L-phenylalanine, $300 \mathrm{ml}$ of abs. EtOH and dry HCl.

L-Aspartic Acid Ethyl Ester Hydrochloride: It was prepared by the method of Fischer ${ }^{5)}$ using $30 \mathrm{~g}$ $(0.226$ mole) of L-aspartic acid, $300 \mathrm{ml}$ of abs. EtOH and dry $\mathrm{HCl}$.

L-Valine Ethyl Ester Hydrochloride: It was prepared by the method of Tafele using $30 \mathrm{~g}(0.26$ mole $)$ of $\mathrm{L}$-valine, $200 \mathrm{ml}$ of abs. EtOH and dry $\mathrm{HCl}$.

General Method for Preparation of $\alpha$-Substituted Amino Acid Esters: To 0.57 mole of ethyl chloroacetate was added 1.14 mole of amines in small portions under cooling with ice and salt. The mixture was allowed to stand overnight at room temperature. Amine hydrochloride precipitated was removed by suction, and the filtrate was diluted with $\mathrm{H}_{2} \mathrm{O}$ and extracted with ether. After removal of ether, the residue was distilled under reduced pressure.

$\boldsymbol{\beta}$-Amino Acid Esters-Method I: By the method of Adamson ${ }^{36}$ ) adding 1 mole of amine to a solution of 0.5 mole of ethyl acrylate in $250 \mathrm{ml}$ of $\mathrm{EtOH}$

Method II : By the method of Flürscheim ${ }^{3 c}$ ) refluxing a mixture of 0.5 mole of ethyl acrylate and 1 mole of amine.

Method III: By the method of Adamson adding a solution of 0.5 mole of ethyl acrylate in $100 \mathrm{ml}$ of abs. EtOH to 1 mole of amine.

Method IV: By the method of Pacheo ${ }^{3 e}$ ) from 0.17 mole of ethyl cinnamate and 0.34 mole of amine.

Method V: A solution of 0.5 mole of ethyl acrylate in $100 \mathrm{ml}$ of EtOH was added to 0.5 mole of amine. The solution was refluxed for $5 \mathrm{hr}$ on a water bath. After removal of EtOH, the residue was extracted with ether, dried over $\mathrm{Na}_{2} \mathrm{SO}_{4}$, and distilled under reduced pressure.

These compounds obtained were summarized in Table II.

4) E. Fischer and W. Schoeller, Ann., 357, 14 (1907).

5) E. Fischer, Chem. Ber., 34, 452 (1901).

6) J. Tafel, Chem. Ber., 22, 1862 (1889). 
TABLE II. Ethyl 2-Substituted Amino-2-alkylpropionate

\begin{tabular}{|c|c|c|c|c|c|c|c|}
\hline $\mathrm{R}_{2} \mathrm{~N}$ & $\mathrm{R}^{\prime}$ & Method & Solvent & $\underset{(\mathrm{hr})}{\mathrm{Time}}$ & $\underset{\left({ }^{\circ} \mathrm{C}\right)}{\text { Tempt. }}$ & $\underset{\left({ }^{\circ} \mathrm{C} / \mathrm{mm}\right)}{\mathrm{bp}} \underset{\left({ }^{\circ} \mathrm{C}\right)}{\text { or }}$ & $\begin{array}{l}\text { Yield } \\
(\%)\end{array}$ \\
\hline $\mathrm{C}_{2} \mathrm{H}_{5} \mathrm{NH}$ & $\mathrm{H}$ & $\mathrm{I}$ & EtOH & 24 & $10-20$ & $101-103 / 17$ & 75 \\
\hline$\left(\mathrm{CH}_{3}\right)_{2} \mathrm{~N}$ & $\mathrm{H}$ & $I$ & $\mathrm{EtOH}$ & 24 & $10-20$ & $65-66 / 24$ & 76 \\
\hline$\left(\mathrm{C}_{2} \mathrm{H}_{5}\right)_{2} \mathrm{~N}$ & $\mathrm{H}$ & II & no & 4 & $50-60$ & $94.5-95.5 / 24$ & 73 \\
\hline$\left(\mathrm{C}_{2} \mathrm{H}_{5}\right)_{2} \mathrm{~N}$ & $\mathrm{CH}_{3}$ & II & no & 4 & $50-60$ & $94-95 / 17.5$ & 11 \\
\hline$\left(\mathrm{C}_{2} \mathrm{H}_{5}\right)_{2} \mathrm{~N}$ & $\mathrm{CH}_{3}$ & I & EtOH & 7 & $10-20$ & $94-95 / 17.5$ & 14 \\
\hline & $\mathrm{H}$ & II & no & 6 & $90-100$ & $93-95 / 2$ & 78 \\
\hline & $\mathrm{Ph}$ & $\mathbb{N}$ & no & 3 & $70-80$ & $\begin{array}{l}135-138 / 3 \\
\text { (171-172 hydrochloride) }\end{array}$ & 14 \\
\hline & $\mathrm{H}$ & II & no & 6 & $100-110$ & $127-128 / 32$ & 88 \\
\hline & $\mathrm{Ph}$ & $\mathbb{N}^{a)}$ & no & 3 & $70-80$ & $\begin{array}{l}205 \\
\text { (hydrochloride) }\end{array}$ & 16 \\
\hline$N$ & $\mathrm{H}$ & II & no & 6 & $100-110$ & $111-112 / 10$ & 82 \\
\hline $\mathrm{PhCH}_{2} \mathrm{NH}$ & $\mathrm{H}$ & III & $\mathrm{EtOH}$ & 5 & $80-85$ & $122-125 / 1.5$ & 43 \\
\hline $\mathrm{N} \rightleftharpoons \mathrm{N}$ & $\mathrm{H} \cdots$ & V & $\mathrm{EtOH}$ & 5 & $70-80$ & $\begin{array}{l}34-35 \\
(211 \text { hydrochloride) }\end{array}$ & $11^{b)}$ \\
\hline
\end{tabular}

a) In this case, 3-phenyl-3-piperidinopropiopiperidide ( $\mathrm{mp} 119-120)$ and cinnamoylpiperidide (mp 122-125) were also isolated.

b) N,N-carboethoxyethylpiperazine hydrochloride: Anal. Calcd. for $\mathrm{C}_{14} \mathrm{H}_{28} \mathrm{O}_{4} \mathrm{~N}_{2} \mathrm{Cl}_{2}:$ C, 46.80; H, 7.85; N, 7.79. Found: C, $47.00 ; \mathrm{H}, 7.90 ; \mathrm{N}, 7.78$.

General Method for Synthesis of Amino-tert-alcohol_-Magnesium turnings (0.145 atom) was covered with $20 \mathrm{ml}$ of ether and a small amount of halide was added with warming to initiate the reaction. If the reaction did not initiate, a catalytic amount of iodine was added. As soon as the reaction was initiated, the remainder of halide (total $0.145 \mathrm{~mole}$ ) was gradually added at such a rate that the mixture boils gently. After addition, the mixture was warmed to complete the reaction. To this solution was added a calculated amount of amino acid ester at such a rate that the reaction mixture boils continuously. The reaction mixture was refluxed for 30 min to complete the reaction, and then treated with $15 \%$ aqueous solution of $\mathrm{HCl}$ containing ice pieces. The insoluble oily substance, mainly hydrccarbon produced by coupling, was removed by extraction with ether. The aqueous layer was made alkaline with aqueous $\mathrm{NH}_{4} \mathrm{OH}$, and extracted with ether. The product was purified by distillation or recrystallization in the form of oxalate.

Acknowledgement The authors are deeply grateful to Miss M. Sato for IR spectral measurements and to K. Ogata for elementary analysis data. 